\title{
Asynchronous Digital SQUID Magnetometer with an On-Chip Magnetic Feedback for Improvement of Magnetic Resolution
}

\author{
Yuto Tsuga, Yuki Yamanashi, and Nobuyuki Yoshikawa, Member, IEEE
}

\begin{abstract}
An asynchronous digital SQUID with complementary output, which needs no periodic high-frequency readout clock input, has been investigated. The magnetic flux resolution of the asynchronous digital SQUID has been improved using an on-chip magnetic feedback. A wide dynamic range asynchronous digital SQUID magnetometer system with improved magnetic resolution has been devised using alternating readout of two digital SQUIDs placed in parallel. The slew rate and the dynamic range of the asynchronous digital SQUID magnetometer were estimated to be $4 \times 10^{10} \Phi_{0} / \mathrm{s}$ and the quite large, respectively. We have designed the asynchronous digital SQUID magnetometer system using $2.5 \mathrm{kA} / \mathrm{cm}^{2} \mathrm{Nb}$ process. We have experimentally confirmed the magnetic flux resolution of less than flux quantum $\left(\Phi_{0}\right)$ and the good linearity of the magnetic response of the asynchronous digital SQUID magnetometer system up to $3000 \Phi_{0}$.
\end{abstract}

Index Terms - SQUID, Digital SQUID, Single flux quantum circuit, Dynamic range

\section{INTRODUCTION}

A SUPERCONDUCTING quantum interference device (SQUID) is used as an extremely high sensitive magnetometer, which is applied in various fields, such as medical diagnoses, material evaluation, and mineral exploration [1-5]. The superconductive magnetic sensors commercially used at present are dc-SQUIDs, which can detect $10^{-4}$ of the flux quantum in a superconductor, $\Phi_{0}$ (= $\left.2.07 \times 10^{-15} \mathrm{~Wb}\right)$. A multi-channel SQUID magnetometer can be used for more effective magnetic measurements in many applications [5-8]. The conventional SQUID magnetometer requires a flux locked loop (FLL) circuit placed at the roomtemperature stage to keep the operating point of the dc-SQUID to be the optimum, where the largest voltage change can be obtained when the measured magnetic field changes, for each channel. Because the number of lines that connect the lowtemperature and the room-temperature stages increases with

Manuscript received October 9, 2012.

This work was supported by "Promotion of Environmental Improvement for Independence of Young Researchers" under the Special Coordination Funds for Promoting Science and Technology from the Ministry of Education, Culture, Sports, Science and Technology (MEXT) of Japan. The National Institute of Advanced Industrial Science and Technology contributed in part to the circuit fabrication.

Y. Tsuga, Y. Yamanashi and N. Yoshikawa are with the Department of Electrical and Computer Engineering, Yokohama National University, Yokohama 240-8501, Japan (corresponding author e-mail: yamanasi@ynu.ac.jp). an increase in the number of SQUID channels, the building a large scale multi-channel SQUID magnetometer is difficult using the conventional SQUIDs. Moreover, the slew rate of the SQUID magnetometer is limited by the bandwidth of the FLL.

In 1980's, a digital SQUID based on the superconductive latching logic circuits have been designed and fabricated [9, 10]. The digital SQUID carries out the digital feedback using a delta modulator. The digital SQUID magnetometers, in which dc-SQUID and superconductive single flux quantum (SFQ) circuits [11] are integrated at the low-temperature stage, have been proposed and implemented [12-14]. In the digital SQUID, SFQ circuits are used to control and measure SFQ pulses outputted by the dc-SQUID sensor. 1-channel digital SQUID based on SFQ logic circuits is similar to analog/digital converter $[15,16]$. The slew rate of the digital SQUID magnetometer, which corresponds to the bandwidth of the measurable magnetic field, is much higher than that of the conventional SQUID due to the high-speed operation of SFQ readout circuits. The digital SQUID magnetometer has the wide dynamic range compared with the conventional SQUID because the magnetic field can be measured by SFQ logic circuits as binary digital data. Moreover, multi-channel SQUID magnetometers can be implemented with the small number of lines which connect the low-temperature circuit to the room-temperature electronics by using the digital SQUID. However, the magnetic flux resolution of the previously proposed digital SQUID was $\Phi_{0}$.

We have proposed an asynchronous digital SQUID with complimentary output [17], which needs no high-frequency clock input for the readout. However, the magnetic flux

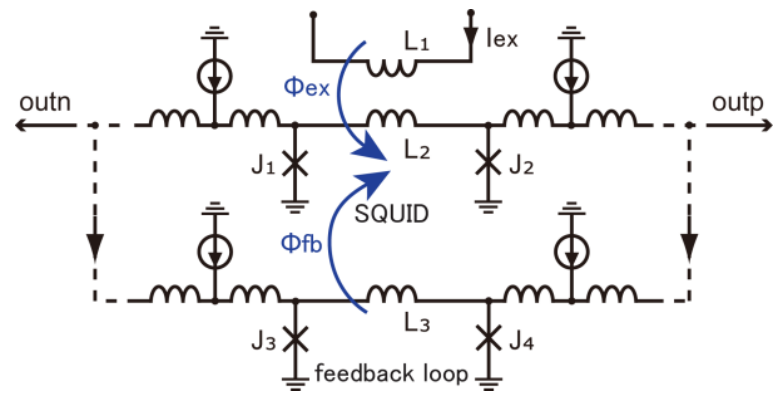

Fig. 1. Equivalent circuit of the asynchronous digital SQUID with an on-chip magnetic feedback. $\mathrm{L}_{1}=15 \mathrm{pH}, \mathrm{L}_{2}=154 \mathrm{pH}, \mathrm{L}_{3}=222 \mathrm{pH}, \mathrm{J}_{1}=$ $\mathrm{J}_{2}=100 \mu \mathrm{A}, \mathrm{J}_{3}=\mathrm{J}_{4}=450 \mu \mathrm{A}$. 


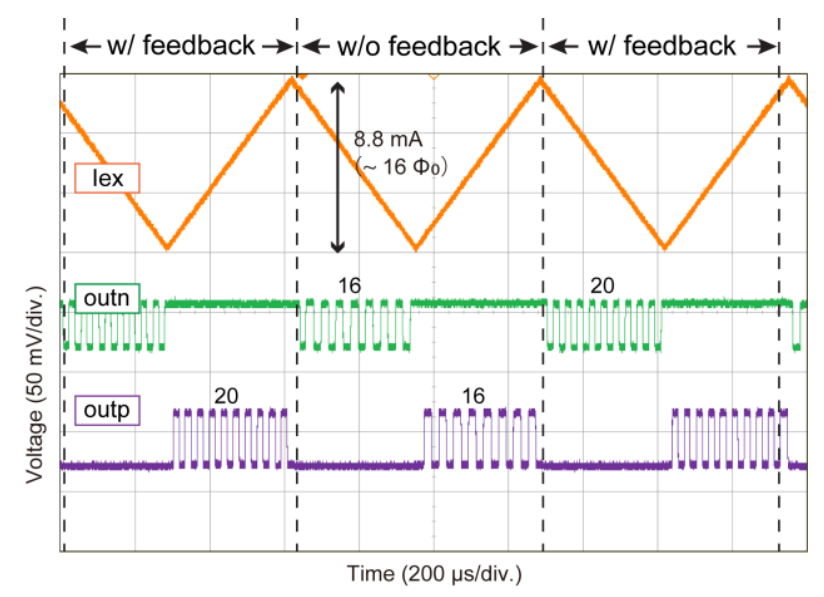

Fig. 2. Measured waveforms of the aynchronous digital SQUID. $I_{e x}$ is corresponds to the input magnetic field $\Phi_{\mathrm{ex}}$. Lower two waveforms are output of the asynchronous digital SQUID, and level transition corresponds to the output of SFQ signals. In the region labeled as "w/ feedback," the on-chip magnetic feedback is applied and the magnetic resolution is improved. In the region labeled as "w/o feedback," the onchip magnetic feedback is not used.

resolution of the asynchronous digital SQUID was still $\Phi_{0}$, though the slew late was improved due to the asynchronous operation. In this study, we have proposed a new asynchronous digital SQUID magnetometer system, the magnetic flux resolution of which is improved to less than $\Phi_{0}$ with the quite large dynamic range. The circuit design and experimental results of the asynchronous digital SQUID system are reported.

\section{ASYNCHRONOUS DIGITAL SQUID}

Fig. 1 shows an equivalent circuit of the asynchronous digital SQUID with magnetic feedback for improving the magnetic resolution. The magnetic flux induced by the external current $I_{e x}$ is input to the SQUID loop. When the magnetic flux applied to the SQUID loop $\Phi_{\mathrm{ex}}$ increases, the current flowing in the Josephson junction $\mathrm{J}_{1}$ is increased by the circulating current induced by the increase of the applied magnetic flux. When the current flowing in $J_{1}$ reaches its critical current, an SFQ pulse is output to the "outp" port without the inputting readout clock. Similarly, SFQ pulses are output to the "outn" port when the $\Phi_{\text {ex }}$ decreases. This operation is similar to the bidirectional ADC modulator [15, 16], however, in this system, high speed operation is possible because of the asynchronous operation. Though asynchronous counters are needed to implement the magnetometer system, the slew rate is not limited by the readout clock frequency. Furthermore, an on-chip magnetic feedback is used to improve the magnetic flux resolution as shown in Fig. 1. SFQ pulses from the SQUID loop are output and input to the magnetic feedback loop simultaneously. Magnetic flux quanta stored in the feedback loop apply magnetic fields to the SQUID loop via the mutual positive coupling between feedback and SQUID loops and induce the circulating current in the SQUID loop. Therefore, the following SFQ output can be obtained with an increase of the input magnetic field less than $\Phi_{0}$. That results in the improvement of the resolution of the magnetic

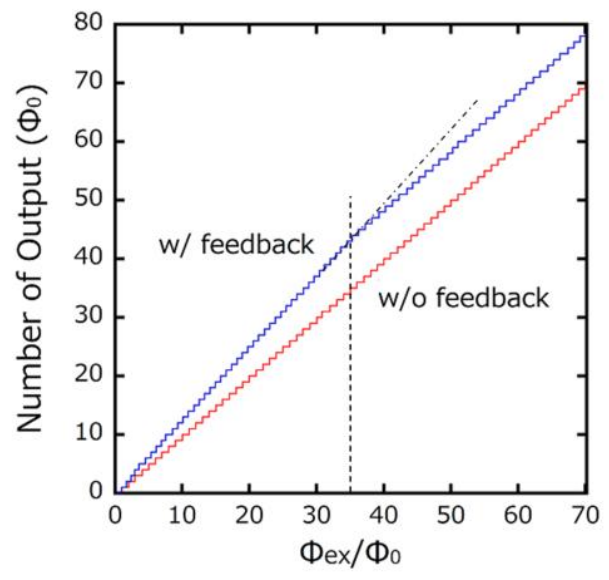

Fig. 3. Dependence of the number of SFQ outputs on the input magnetic flux amplitude. The characteristic labeled as "w/ feedback" corresponds to the measured result when the on-chip magnetic feedback is used. The slopes of the characteristics correspond to the magnetic flux resolution. Resolutions of "w/o feedback" and "w/ feedback" are $\Phi_{0}$ and $0.8 \Phi_{0}$, respectively. The liniarity up to $35 \Phi_{0}$ was measured with the improved magnetic flux resolution.

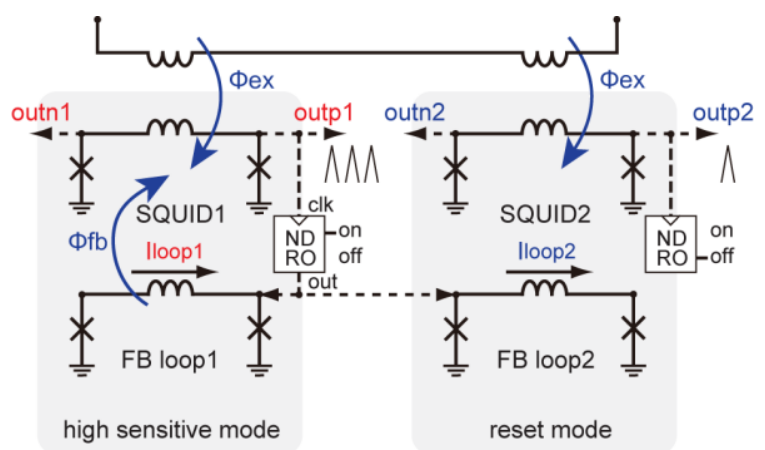

(a)

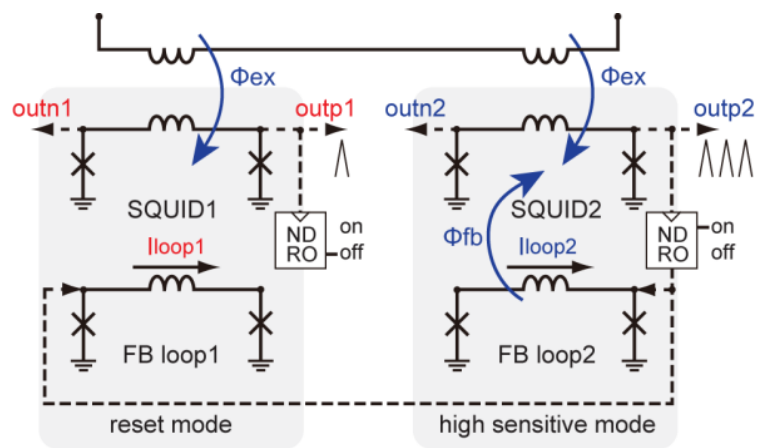

(b)

Fig. 4. Concept of the high-sensitive asynchronous digital SQUID magnetometer with the wide dynamic range. Two operating mode, represented by (a) and (b), where one asynchronous digital SQUID is in high-sensitive mode by magnetic feedback and the other SQUID is in the reset mode, are repeated alternately when the input magnetic flux continue to increase or decrease. NDRO (Non-destructive read-out) gates correspond to SFQ switches.

flux. The improved magnetic flux resolution depends of the strength of the magnetic coupling between feedback and SQUID loops. The change of the input magnetic flux $\Phi_{\text {ex }}$ can be measured by integrating the number of asynchronous SFQ output.

Fig. 2 shows a measurement result of the asynchronous 


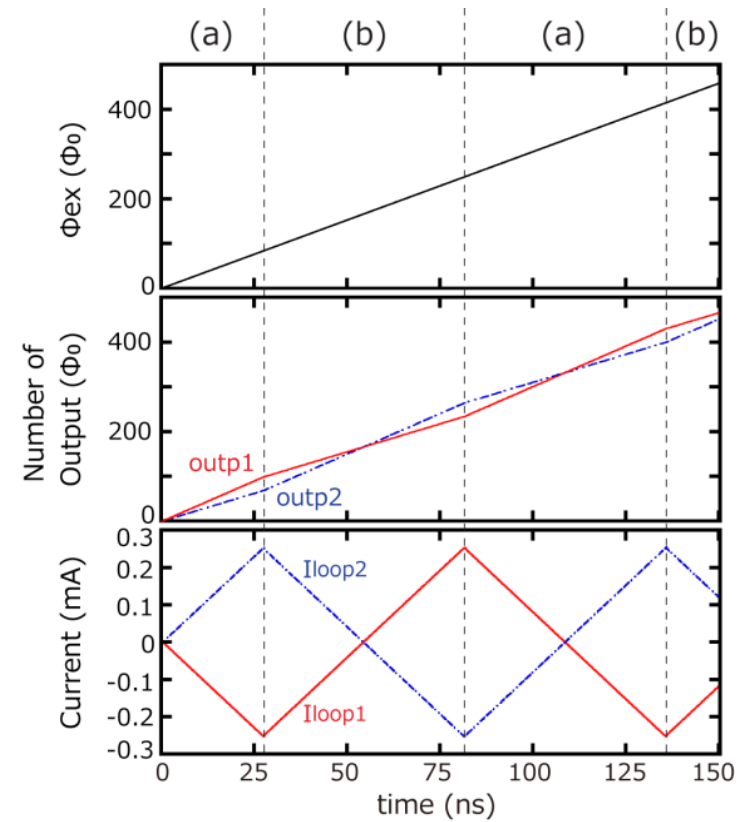

Fig. 5. Simulation result of the high-sensitive asynchronous digital SQUID magnetometer with the wide dynamic range. Two operations shown in Fig. 4 are alternately changed at dashed lines.

digital SQUID with the improved magnetic flux resolution implemented by using the ISTEC-SRL $10 \mathrm{kA} / \mathrm{cm}^{2} \mathrm{Nb}$ advanced process [18]. In this test circuit, the magnetic coupling coefficient between feedback and SQUID loops was 0.4 and the on-chip magnetic feedback can be turned on and off. In this measurement, the magnetic flux of $\pm 8 \Phi_{0}$ was inputted by $\mathrm{I}_{\mathrm{ex}}$. In the "w/o feedback" region, where magnetic feedback was not used, the number of output pulses was 16. It indicated the magnetic resolution of the digital SQUID without the magnetic feedback was $\Phi_{0}$. As shown in Fig. 2, the number of output SFQ pulses was increased from 16 to 20 by turning the on-chip magnetic feedback on. In this measurement result, the magnetic flux resolution was improved to $16 / 20 \Phi_{0}=0.8 \Phi_{0}$. Because this operation is completely asynchronous, the slew rate of the asynchronous digital SQUID is limited by a time required for the magnetic feedback. Assuming the ISTEC-SRL $10 \mathrm{kA} / \mathrm{cm}^{2} \mathrm{Nb}$ advanced process, the time required for the magnetic feedback is estimated to be 20 ps. Therefore, the slew rate of the asynchronous with improved magnetic flux resolution is estimated to be about $0.8 \Phi_{0} / 20 \mathrm{ps}=4 \times 10^{10} \Phi_{0} / \mathrm{s}$.

However, the dynamic range of the high-sensitive asynchronous digital SQUID was limited by the maximum number of flux quanta that can be stored in the feedback loop. In this test circuit, the number of flux quanta can be stored in the feedback loop was 35. Fig. 3 shows a measured dependence of the number of SFQ output from the asynchronous digital SQUID on the amplitude of input magnetic flux. The good linearity up to $3000 \Phi_{0}$ has been experimentally confirmed when the magnetic feedback was not used [17]. On the other hand, the linearity of up to only 35 $\Phi_{0}$ was obtained in the case of using the on-chip magnetic feedback. Accordingly, the dynamic range of this high-

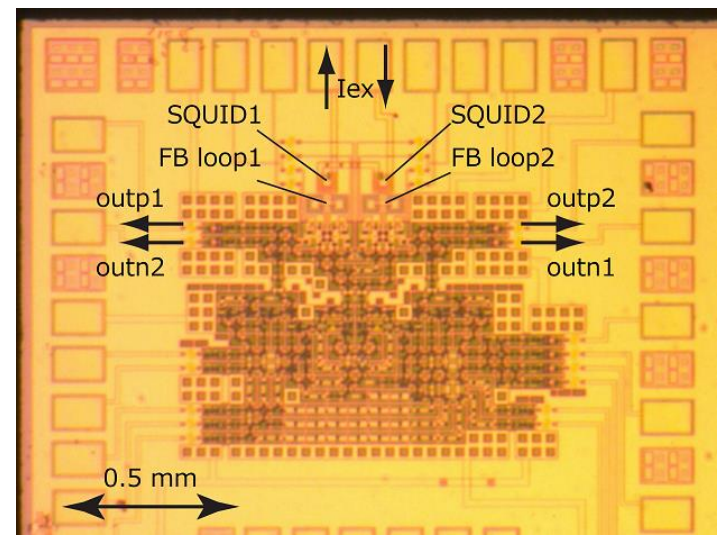

Fig. 6. Chip photograph of the high-sensitive asynchronous digital SQUID magnetometer system

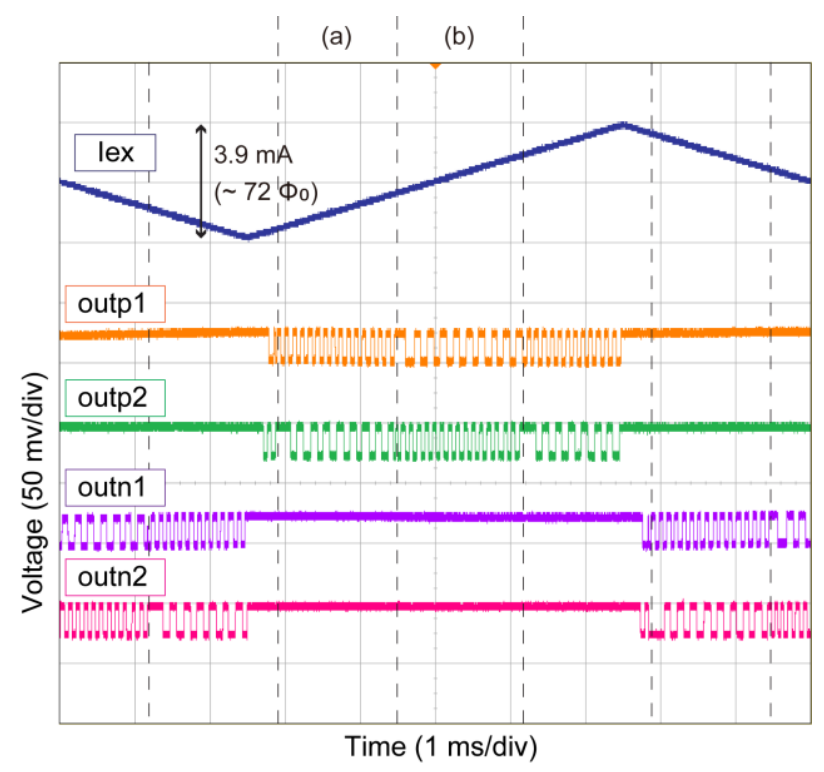

Fig. 7. Measured waveforms of the wide dynamic range asynchronous digital SQUID system. Two operations, correspoding to Fig. 4 (a) and (b), are switched alternately dashed lines.

sensitive asynchronous digital SQUID using the on-chip magnetic feedback was $35 \Phi_{0}$. This value is not enough for practical magnetometer. To improve the small dynamic range of the asynchronous digital SQUID, we have devised the new digital SQUID magnetometer system.

\section{High-SENSITIVE ASYNCHRONOUS DigitAL SQUID MAGNETOMETER SYSTEM WITH WIDE DYNAMIC RANGE}

Fig. 4 illustrates a basic concept of the high-sensitive asynchronous digital SQUID magnetometer system with the wide dynamic rage. In this system, two asynchronous digital SQUIDs, mentioned in the previous section, are used. The magnetic feedback is turned on and off by controlling SFQ switches that control the propagation of SFQ pulses. While the one digital SQUID is set to be in the high-sensitive mode by turning the magnetic feedback on, the other digital SQUID is reset by inputting SFQ pulses to the other side of input port to erase the flux quanta stored in the feedback loop. When the input magnetic flux reaches its dynamic range of the 


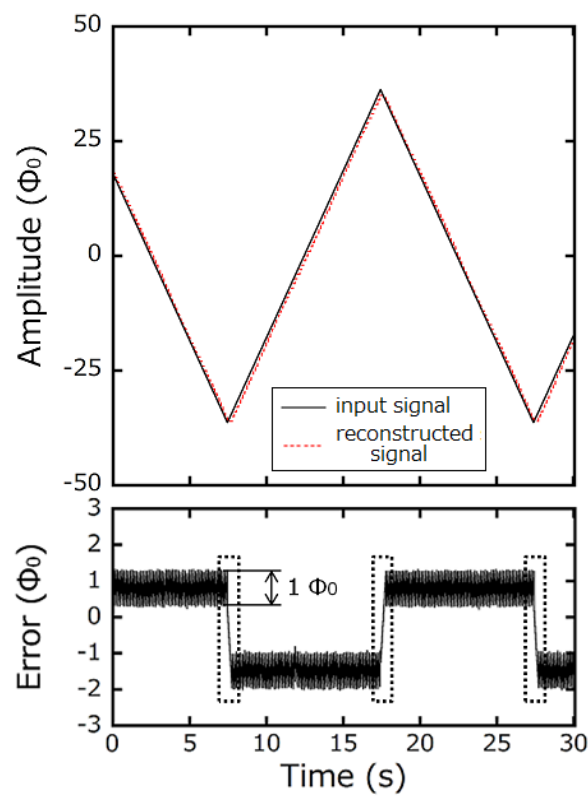

(a)

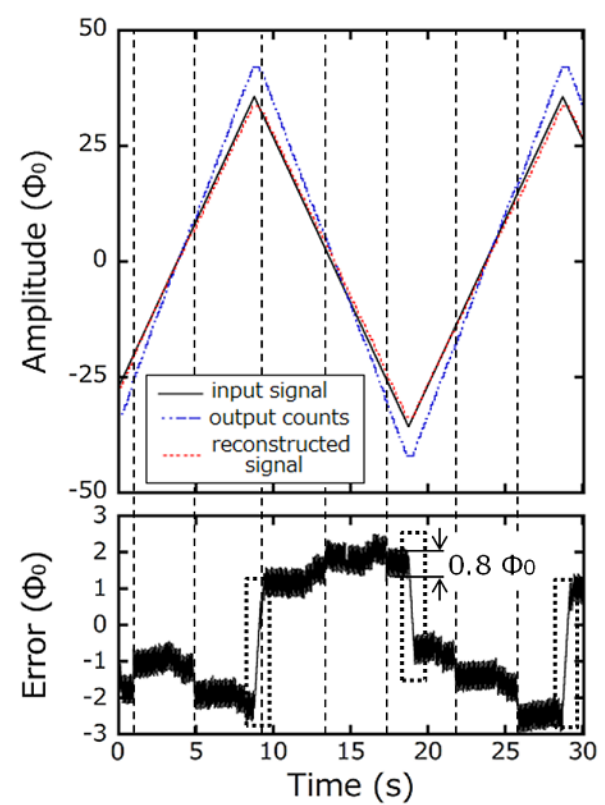

(b)

Fig. 8. Comparison of input and reconstructed waveforms. Results of (a) the asynchronous digital SQUID without the on-chip magenetic feedback and (b) high-sensitive asynchronous digital SQUID magnetometer system. Two operations, correspoding to Fig. 4 (a) and (b), are switched alternately at the time represented by dashed lines in (b). The "output counts" is output data before normalization, and the "reconstructed signal" is normalized waveform in (b).

asynchronous digital SQUID in the high-sensitive mode, the operating mode switches to its reset mode by detecting the overflowed SFQ pulse from the feedback loop. The other digital SQUID is set to the high-sensitive mode simultaneously. In this system, the dynamic range appears unlimited, but is in fact limited by magnetic fields.

Fig. 5 shows a simulation result of the asynchronous digital SQUID magnetometer system. Two operations, represented by (a) and (b) in Fig. 4, are repeated alternately when the large magnetic flux is input. By counting the output SFQ pulses from the asynchronous digital SQUID in the high-sensitive mode, the output signal with the improved magnetic resolution can be obtained.

We have designed and implemented the proposed asynchronous digital SQUID magnetometer system using the $2.5 \mathrm{kA} / \mathrm{cm}^{2} \mathrm{Nb}$ standard process [19]. Fig. 6 shows a chip photograph of the test circuit containing 645 Josephson junctions. We have input the triangle magnetic flux, the amplitude of which was $35 \Phi_{0}$, and tested the asynchronous digital SQUID magnetometer system. Fig. 7 shows input and measured output waveforms. In this test circuit, SFQ output from both of two asynchronous digital SQUID were observed to monitor the internal operation of the magnetometer. As shown in Fig. 7, the alternating switching of the two operations represented by Fig. 4 (a) and (b) was confirmed.

We processed output data from the digital SQUID system and reconstructed the input magnetic flux. Output data were measured by an automated measurement system and reconstructed waveforms were obtained in the real time. Fig. 8 shows the comparison of input and reconstructed waveforms from output data. The error, the difference between input and reconstructed waveforms, are also shown. Sharp transitions of errors represented by dashed rectangles are caused by the hysteresis of the input-output characteristic of the SQUID loop. This phenomenon was discussed in detail by Haverkamp et al. as the dead zone of the digital SQUID magnetometer [14]. These sharp transitions of the error can be deleted by careful circuit design and adjustment of the bias current supplied to the SQUID loop. The good linearity was confirmed also from the error with a constant quantization level of $\Phi_{0}$ in the asynchronous digital SQUID without the feedback (Fig. 8 (a)). As shown in Fig. 8 (b), the quantization level, that is, the magnetic resolution is $0.8 \Phi_{0}$. However, the quantization level has shifted at the time of switches of operation mode. Even though the error contains it caused by the dead zone and the switch of operation mode, we have confirmed that the input magnetic flux was reconstructed with the error of less than \pm 3 $\Phi_{0}$ using the high-sensitive asynchronous digital SQUID magnetometer system.

\section{CONCLUSION}

We have investigated the high-sensitive asynchronous digital SQUID with complementary output. Due to the asynchronous operation, the slew rate is higher than that of conventional synchronous digital SQUIDs. We have devised the asynchronous digital SQUID magnetometer system, which has wide dynamic range, by using alternating readout of two high-sensitive asynchronous digital SQUIDs. We have implemented and tested the high-sensitive asynchronous digital SQUID magnetometer system. The high-sensitive operation and the wide dynamic ranges were experimentally confirmed. We demonstrated that a large input magnetic signal can be reconstructed by using the high-sensitive asynchronous digital SQUID magnetometer system. 


\section{REFERENCES}

[1] T. Nagaishi, H. Ota, E. Arai, T. Hayashi, and H. Itozaki, "High Tc SQUID system for transient electromagnetic geophysical exploration," IEEE Trans. Appl. Supercond., vol. 15, pp. 749-752, Jun. 2005.

[2] S. Linzen, A. Chwala, V. Schultze, M. Schulz, T. schuler, R. Stolz, N. Bondarenko, and H. G. Meyer, "A LTS-SQUID System for Archaeological Prospection and Its Practical Test in Peru," IEEE Trans. Appl. Supercond., vol. 17, pp. 750-755, Jun. 2007.

[3] S. Keenan, E. J. Romans, and G. B. Donaldson, "HTS SQUID NDE of Curved Surfaces Using Background Field Cancellation Techniques," IEEE Trans. Appl. Supercond., vol. 17, pp. 784-787, Jun. 2007.

[4] Y. Hatsukade, A. Kosugi, K. Mori, and S. Tanaka, "Detection of micro flaws on thin copper tubes using SQUID-NDI system based on eddy current technique," Physica C, vol. 426-431, pp. 1585-1590, Feb. 2005.

[5] K. Nakayama, Y. Kaneda, A. Hirano, and Y. Haruta, "A BCI using MEGvision and multilayer neural network -channel optimization and main lobe contribution analysis-," IEEE ISPACS 2008, Bankok, Thailand, pp. 316-319, Feb. 2009.

[6] I. D. Voitovych, M. A. Primin, and V. N. Sosnytskyy, "Application of SQUIDs for registration of biomagnetic signals," Low Temp. Phys. vol. 38, pp. 311-321, Apr. 2012.

[7] M. J. Chiu, H. E. Horng, J. J. Chieh, S. H. Liao, C. H. Chen, B. Y. Shih, C. C. Yang, C. L. Lee, T. F. Chen, S. Y. Yang, C. Y. Hong, and H. C. Yang, "Multi-Channel SQUID-Based Ultra-High-Sensitivity In-Vitro Detections for Bio-Markers of Alzheimer's Disease Via Immunomagnetic Reduction," IEEE Trans. Appl. Supercond., vol. 21, pp.477-480, Jun. 2011.

[8] V. S. Zotev, A. N. Matlachov, P. L. Volegov, H. J. Sandin, M. A. Wspy, J. C. Mosher, A. V. Urbaitis, S. G. Newman, and R. H. Kraus Jr., "Multi-Channel SQUID System for MEG and Ultra-Low-Field MRI," IEEE Trans. Appl. Supercond., vol. 17, pp.839-842, Jun. 2007.
[9] N. Fujimaki, H. Tamura, T. Imamura, and S. Hasuo, "A Single-Chip SQUID Magnetometer," IEEE Trans. Electron. Devices, ED-35, pp. 2412-2418, Dec. 1988.

[10] U. Fath, R. Hundhausen, T. Fregin, P. Gerigk, W. Eschner, A. Schindler, and F. H. Uhlmann, "Experimental Digital SQUID with Integrated Feedback Circuit," IEEE Trans. Appl. Supercond., vol. 7, pp. 2747-2751, Jun. 1997.

[11] R. P. Robertazzi and S. V. Rylov, "Synchronous Flux Quantizing Analog-to-Digital-Converter," IEEE Trans. Appl. Supercond., vol. 3, pp. 3114-3116, Dec. 1993.

[12] O. Mukhanov, D. Gupta, A. Kadin, and V. Semenov, "Superconductor Analog-to-Digital Converters," Proc. IEEE, vol. 92, pp. 1564-1584, Oct. 2004.

[13] K. K. Likharev and V. K. Semenov, "RSFQ Logic/memory family: A new Josephson-junction technology for sub-terahertz-clock-frequency digital systems," IEEE Trans. Appl. Supercond., vol. 1, pp. 3-28, Mar. 1991.

[14] V. K. Semenov, "Digital SQUIDs: New Definitions and Results," IEEE. Trans. Appl. Supercond., vol. 13, pp. 747-750, Jun. 2003.

[15] T. Reich, T. Ortlepp, and F. Uhlmann, "Digital SQUID Sensor Based on SFQ Technique," IEEE Trans. Appl. Supercond., vol. 15, pp.3-28, Jun. 2005.

[16] I. Haverkamp, O. Mielke, J. Kunert, R. Stolz, H. G. Meyer, H. Toepfer, and T. Ortlepp, "Linearity of a Digital SQUID Magnetometer," IEEE Trans. Appl. Supercond., vol. 21, pp. 705-708, Jun. 2011.

[17] Y. Tsuga, Y. Yamanashi, and N. Yoshikawa, "Development of an Asynchronous Digital SQUID Magnetometer," 6th EASSE, Yonezawa, Japan, Oct. 2011.

[18] S. Nagasawa, K. Hinode, T. Satoh, H. Akaike, Y. Kitagawa, and M. Hidaka, "Development of advanced Nb process for SFQ circuits," Physica C, vol. 412-414, pp. 1429-1436, Oct. 2004.

[19] S. Nagasawa, Y. Hashimoto, H. Numata, and S. Tahara, "A 380 ps. 9.5 $\mathrm{mW}$ Josephson 4-kbit RAM operated at a high bit yield," IEEE Trans. Appl. Supercond., vol. 5, pp. 2447-2452, Jun. 1995. 